and constant increase in CDI hospitalization and mortality rates.

Methods To describe the epidemiology of CDI hospitalization in Veneto Region, a retrospective analysis based on the anonymous regional database was carried out including all discharges of the period 200-016 of population over 65 years of age with a code for CDI (008.45) as principal or secondary diagnosis.

Data were stratified by Major Diagnostic Categories (MDC), which are formed by dividing all ICD-9-CM diagnoses into 25 mutually exclusive diagnosis areas, to evaluate the in-hospital mortality risk attributable to CDI of each MDC.

Results 6640 discharges with CDI in patients over 65 years of age were tracked, with an overall lenght of stay (LOS) of $25.6 \pm 22,7$ days. Hospitalization distribution by gender showed a prevalence of females $(63.2 \%)$ characterized by a higher mean age $(80.2 \pm 14.6$ Vs. $75.6 \pm 16.4$ yrs;p<0.001). The rate of CDI diagnoses per 100000 overall admissions increased steeply with age, and reached a peak of 222.4 every 100000 discharges among the very elderly ( $>$ 85years). In the frame of over 65 years patients the age class distribution showed a higher risk in $7-4$ (OR:1.62; $<0.001)$ and an almost double in $>85$ years $(\mathrm{OR}: 2.1 ; \mathrm{p}<0.001)$ respect to $6-4$ age class. Over 65 years of age patients admission with CDI diagnosis represent $2.1 \%$ of overall ordinary admission and cause to the LOS excess of 15.7 days and represent the 5.4\%o of overall hospitalization days. Also in-hospital mortality rate showed an excess risk attributable to CDI (OR:1.91;p<0.001). As expected the MDC with most discharges of CDI was n.6 "Digestive"(36\%) followed by 4 "Respiratory" (17\%). The higher excess of in-hospital mortality risk was a prerogative of n. 8 "Musculoskeletal"(OR:5.55;p<0.001) followed by 11 "Kidney And Urinary Tract" (OR:3.6;p<0.001) and 5 "Circulatory"(OR:2.35; p<0.001). Otherwise the presence of CDI showed a slight excess risk in MDC n.6 "Digestive"(OR:1.03; $\mathrm{p}<0.05), 18$ "Infectious and Parasitic"(OR:1.05;p<0.001) and 4 "Respiratory"(OR:1.01; $<0.001)$.

Conclusion In the observation period the standard hospitalization rate for CDI was almost doubled and standard of mortality rate even increased sixfold, with an increase in their frequency, as well as in their severity and in the additional days of hospitalization.

Moreover the stratification of CDI by MDC showed a serious scenary in patients affected by musculoskeletal, kidney and circulatory diseases with an excess of in-hospital mortality risk associated to CDI.

\section{PWE-044 ACUTE COLON DIVERTICULITIS HOSPITALIZATION TRENDS IN VENETO REGION (NORTHEAST ITALY)}

${ }^{1}$ Mario Saia, ${ }^{1}$ Diego Caroli*, ${ }^{1}$ Erik Rosa-Rizzotto, ${ }^{2}$ Claudio Pilerci, ${ }^{2}$ Franca De Lazzari. ${ }^{1}$ St. Anthony Hospital, Padova, Italy; ${ }^{2}$ Azienda Zero, Padova, Italy

10.1136/gutjnl-2019-BSGAbstracts.368

Introduction Diverticular disease (DD) of the colon represents a common clinical condition affecting with high rate the population in developed countries. The rates of hospital admissions for Acute Colon Diverticulitis (ACD), the most important complication of DD, is progressively increased. The aim of our study is to describe the hospitalization trend for ACD in Veneto region, including in-hospital mortality, and the impact of specialistic admission hospital units as Gastroenterologic (GE) units.

Methods Data were obtained from the Veneto Region anonymous Hospital Discharge Record database from 2000 to 2017, in which diverticulitis of colon [ICD-9-CM code 562.11 and 562.13 (diverticulitis with and without mention of haemorrhage)] was the primary diagnosis.

Standardized Hospitalization Rate (SHR) per five-year group was calculated and expressed per 100,000 population. To evaluate the impact of admission hospital units on the outcome of ACD management we analyzed in-hospital mortality, need for surgery (NFS) and lenght of stay (LOS).

Results From 2000 to 2017 a total of 33,249 hospitalization for $\mathrm{AD}$ were registered with an increasing rate from 0.30 to 0.46 for 1,000 inhabitants $\left(\mathrm{X}^{2}\right.$ trend:514.182, $\mathrm{p}<0.001$; mean increase of $2.6 \%$ per year,p $<0.001)($ FIG.1). Mean age was 66.1 \pm 28.4 , higher in female $(70.1 \pm 29.8$ Vs.60.5 \pm 24.9 ; $\mathrm{p}<0.05$ ) and the higher hospitalization rate (overall mean:0.39) was in female (0.43Vs.0.34;OR:1.30;CI95\%:1.2$.32 ; \mathrm{p}<0.001)$. SHR increased with age reaching the higher value in patients $\geq 85$ years (162.43) with an increase of hospitalization over the study period for all class ages $(p<0.001)$. Female showed an higher prevalence of hospitalization compared to men (OR:1.30;CI95\%: 1.2-.32;p<0.001), and both genders presented an increasing trend, with a mean increase per year of $1.9 \%$ and $3.8 \%$ respectively $(p<0.001)$. NFS was substantially stable (19.4\%) while overall in-hospital mortality rate showed an important increase from 0.37 to 0.98 $(\mathrm{p}=0.037)$. Also LOS decreased significantly $(-18 \%)$ from 9.8 to 8 days $(p<0.001) .73 \%$ of patients were overall admitted by surgical units with a steady increase for admission in medical ones from $22 \%$ to $34 \%$ and in particular in GE units where admission rose from $7 \%$ to $22 \%$ of overall admission of Medical Area. From the comparison between GE units and other medical area admissions, emerged that patients admitted in GE units have a not significantly lower $(p>0.05)$ NFS rate (3.5\%Vs.4.1\%) and in-hospital mortality (0.3\%Vs.1.4\%), showing a significative lower LOS $(7.1 \pm 2.2$ Vs. $11 \pm 3.6 ; \mathrm{p}<0.001)$.

Conclusions Results suggest that ACD is an emerging heathcare problem related to the rising of age. Through the years the NFS rate was stable with an increase of mortality. In this scenary GE units seem to be more efficiently obtaining a significative reduction of the cost management of ACD.

\begin{tabular}{|c|c|c|c|c|c|}
\hline Area & Total & $\begin{array}{l}\% \\
\text { distrib }\end{array}$ & $\begin{array}{l}\% \\
\text { NFS }\end{array}$ & LOS & $\begin{array}{l}\% \\
\text { death }\end{array}$ \\
\hline Surgery & 24323 & $73 \%$ & $25 \%$ & 8,3 & $0,5 \%$ \\
\hline Medical & 8893 & $27 \%$ & $4 \%$ & 10,2 & $1,1 \%$ \\
\hline Intensive Therapy & 33 & $0,1 \%$ & $48 \%$ & 22,8 & $24,2 \%$ \\
\hline Total & 33249 & $100 \%$ & $19 \%$ & 8,8 & $0,6 \%$ \\
\hline $\begin{array}{l}\text { Medical area } \\
\text { only }\end{array}$ & Total & $\begin{array}{l}\% \\
\text { distrib }\end{array}$ & & & \\
\hline Gastro units & 1743 & $20 \%$ & $3,5 \%$ & 7,1 & $0,3 \%$ \\
\hline No Gastro units & 7150 & $80 \%$ & $4,1 \%$ & 11,0 & $1,4 \%$ \\
\hline
\end{tabular}

